\title{
IDEAL EXTENSIONS OF ORDERED SETS
}

\author{
NIOVI KEHAYOPULU
}

Received 30 January 2003

\begin{abstract}
The ideal extensions of semigroups-without order-have been first considered by Clifford (1950). In this paper, we give the main theorem of the ideal extensions for ordered sets. If $P, Q$ are disjoint ordered sets, we construct (all) the ordered sets $V$ which have an ideal $P^{\prime}$ which is isomorphic to $P$, and the complement of $P^{\prime}$ in $V$ is isomorphic to $Q$. Conversely, we prove that every extension of an ordered set $P$ by an ordered set $Q$ can be so constructed. Illustrative examples of the main theorem in case of finite ordered sets are given.
\end{abstract}

2000 Mathematics Subject Classification: 06A06.

1. Introduction and definitions. The extension problem for groups is as follows: given two groups $H$ and $K$, construct all groups $G$ which have a normal subgroup $N$ such that $N$ is isomorphic to $H$ (in symbol, $N \approx H$ ) and $G / N \approx K$ (where $G / N$ is the quotient of $G$ by $N)$. $G$ is called the Schreier's extension or simply the extension of $H$ by $K$. Ideal extensions of semigroups have been considered by Clifford in [3] with exposition of the theory appearing in $[4,10]$. Ideal extensions of totally ordered semigroups have been studied in [6, 7], and the ideal extentions of topological semigroups in [2, 5]. Ideal extensions of lattices have been considered in [8]. Ideal extensions of ordered semigroups have been studied in [9]. The aim of this paper is to construct the ideal extensions of ordered sets. We are often interested in building more complex semigroups, lattices, ordered sets, and ordered or topological semigroups out of some of "simpler" structure and this can be sometimes achieved by constructing the ideal extensions. If $P$ and $Q$ are two disjoint ordered sets, an ordered set $V$ is called an ideal extension (or just an extension) of $P$ by $Q$ if there exists an ideal $P^{\prime}$ of $V$ which is isomorphic to $P$ and the complement $V \backslash P^{\prime}$ of $P^{\prime}$ to $V$ is isomorphic to $Q$. We give the main theorem of such extensions, which is the following: if $\left(P, \leq_{P}\right)$ and $\left(Q, \leq_{Q}\right)$ are two disjoint ordered sets, $r$ an arbitrary subset of $P \times Q$, and

$$
\bar{r}:=\left\{(a, b) \in P \times Q \mid \exists\left(a^{\prime}, b^{\prime}\right) \in r \text { such that } a \leq_{P} a^{\prime}, b^{\prime} \leq_{Q} b\right\},
$$

then the set $V:=P \cup Q$ endowed with the order " $\leq$ " defined by $\leq:=\leq_{P} \cup \leq_{Q} \cup \bar{r}$ is an ordered set and it is an extension of $P$ by $Q$. Conversely, we prove that every extension of $P$ by $Q$ can be so constructed. Some further results and applications of the main theorem to finite ordered sets are also given.

Let $(V, \leq)$ be an ordered set. A nonempty subset $P^{\prime}$ of $V$ is called an ideal of $V$ if $a \in P^{\prime}$ and $V \ni b \leq a$ implies $b \in P^{\prime}[1]$.

Each nonempty subset $P^{\prime}$ of an ordered set $\left(V, \leq_{V}\right)$ with the relation " $\leq_{P^{\prime}}$ " on $P^{\prime}$ defined by $\leq_{P^{\prime}}:=\leq_{V} \cap\left(P^{\prime} \times P^{\prime}\right)$ is an ordered set. In the following, each subset $P^{\prime}$ of 
an ordered set $\left(V, \leq_{V}\right)$ is considered as an ordered set endowed with the order $\leq_{P^{\prime}}:=$ $\leq_{V} \cap\left(P^{\prime} \times P^{\prime}\right)$. We denote by $V \backslash P^{\prime}$ the complement of $P^{\prime}$ in $V$.

Definition 1.1. Let $\left(P, \leq_{P}\right),\left(Q, \leq_{Q}\right)$ be ordered sets and $P \cap Q=\varnothing$. An ordered set $\left(V, \leq_{V}\right)$ is called an ideal extension (or just an extension) of $P$ by $Q$ if there exists an ideal $P^{\prime}$ of $V$ such that

$$
\left(P^{\prime}, \leq_{P^{\prime}}\right) \approx\left(P, \leq_{P}\right), \quad\left(V \backslash P^{\prime}, \leq_{V \backslash P^{\prime}}\right) \approx\left(Q, \leq_{Q}\right)
$$

where $\leq_{P^{\prime}}:=\leq_{V} \cap\left(P^{\prime} \times P^{\prime}\right)$ and $\leq_{V \backslash P^{\prime}}:=\leq_{V} \cap\left(\left(V \backslash P^{\prime}\right) \times\left(V \backslash P^{\prime}\right)\right)$.

Notation 1.2. If $\left(V, \leq_{V}\right)$ is an extension of $P$ by $Q$, we always denote by $\varphi$ and $\psi$ the isomorphisms

$$
\begin{aligned}
\varphi:\left(P, \leq_{P}\right) & \rightarrow\left(P^{\prime}, \leq_{V} \cap\left(P \times P^{\prime}\right)\right), \\
\psi:\left(Q, \leq_{Q}\right) & \rightarrow\left(V \backslash P^{\prime}, \leq_{V} \cap\left(\left(V \backslash P^{\prime}\right) \times\left(V \backslash P^{\prime}\right)\right)\right),
\end{aligned}
$$

respectively.

An extension $V$ of $P$ by $Q$ is also denoted by

$$
V\left(P, Q, \varphi: P \longrightarrow P^{\prime}, \psi: Q \longrightarrow V \backslash P^{\prime}\right)
$$

Notation 1.3. For every $r \subseteq P \times Q$, we always denote by $\bar{r}$ the set defined by (1.1). Clearly, $r \subseteq \bar{r}$.

\section{The main theorem}

THEOREM 2.1. Let $\left(P, \leq_{P}\right),\left(Q, \leq_{Q}\right)$ be ordered sets such that $P \cap Q=\varnothing$. Let $r \subseteq P \times Q$ and $V:=P \cup Q$. Define a relation " $\leq$ " on $V$ as follows:

$$
\leq:=\leq_{P} \cup \leq_{Q} \cup \bar{r}
$$

Then $(V, \leq)$ is an ordered set and it is an extension of $P$ by $Q$. Conversely, let $\left(V, \leq_{V}\right)$ be an extension of $P$ by $Q$. Suppose that there exists an $r \subseteq P \times Q$ such that for the set $\bar{r}$ defined above,

$$
\bar{r}=\left\{(a, b) \in P \times Q \mid \varphi(a) \leq_{V} \psi(b)\right\} .
$$

Then the set $P \cup Q$ endowed with the relation " $\leq$ " defined by $\leq:=\leq_{P} \cup \leq_{Q} \cup \bar{r}$ is an ordered set and $(P \cup Q, \leq) \approx\left(V, \leq_{V}\right)$. 
Proof. (I) The set $(V, \leq)$ is an ordered set. In fact let $a \in V$. If $a \in P$, then $(a, a) \in$ $\leq_{P} \subseteq \leq$. If $a \in Q$, then $(a, a) \in \leq_{Q} \subseteq \leq$. Let $(a, b) \in \leq$ and $(b, c) \in \leq$. Then $(a, c) \in \leq$. Indeed we consider the following cases:

(1) $(a, b) \in \leq_{P},(b, c) \in \leq_{P}$,

(2) $(a, b) \in \leq_{P},(b, c) \in \leq_{Q}$,

(3) $(a, b) \in \leq_{p},(b, c) \in \bar{r}$,

(4) $(a, b) \in \leq_{Q},(b, c) \in \leq_{P}$,

(5) $(a, b) \in \leq_{Q},(b, c) \in \leq_{Q}$,

(6) $(a, b) \in \leq_{Q},(b, c) \in \bar{r}$,

(7) $(a, b) \in \bar{r},(b, c) \in \leq_{P}$,

(8) $(a, b) \in \bar{r},(b, c) \in \leq_{Q}$,

(9) $(a, b) \in \bar{r},(b, c) \in \bar{r}$.

We prove the above-mentioned cases as follows.

(1) If $(a, b) \in \leq_{P},(b, c) \in \leq_{P}$, then $(a, c) \in \leq_{P} \subseteq \leq$.

(2) If $(a, b) \in \leq_{P},(b, c) \in \leq_{Q}$, then $b \in P \cap Q$. The case is impossible.

(3) Let $(a, b) \in \leq_{P},(b, c) \in \bar{r}$. Since $(b, c) \in \bar{r}$, we have $(b, c) \in P \times Q$ and there exists $\left(b^{\prime}, c^{\prime}\right) \in r$ such that $b \leq_{P} b^{\prime}, c^{\prime} \leq_{Q} c$. Since $(a, c) \in P \times Q,\left(b^{\prime}, c^{\prime}\right) \in r, a \leq_{P} b^{\prime}, c^{\prime} \leq_{Q} c$, we have $(a, c) \in \bar{r} \subseteq \leq$.

(5) If $(a, b) \in \leq_{Q},(b, c) \in \leq_{Q}$, then $(a, c) \in \leq_{Q}$.

(8) Let $(a, b) \in \bar{r},(b, c) \in \leq_{Q}$. Since $(a, b) \in \bar{r}$, we have $(a, b) \in P \times Q$ and there exists $\left(a^{\prime}, b^{\prime}\right) \in r$ such that $a \leq_{P} a^{\prime}, b^{\prime} \leq_{Q} b$. Since $(a, c) \in P \times Q,\left(a^{\prime}, b^{\prime}\right) \in r, a \leq_{P} a^{\prime}, b^{\prime} \leq_{Q} c$, we have $(a, c) \in \bar{r} \subseteq \leq$.

The cases (4), (6), (7), (9) are impossible since $P$ and $Q$ are disjoint.

Let $(a, b) \in \leq$ and $(b, a) \in \leq$. Then $a=b$. We put $a$ instead of $c$ in conditions (1), (2), (3), (4), (5), (6), (7), (8), (9) above.

(1) If $(a, b) \in \leq_{P},(b, a) \in \leq_{P}$, then $a=b$.

(2) If $(a, b) \in \leq_{P},(b, a) \in \leq_{Q}$, then $a \in P \cap Q$. The case is impossible.

(3) Let $(a, b) \in \leq_{P},(b, a) \in \bar{r}$. Since $\bar{r} \subseteq P \times Q$, we have $a \in P \cap Q$. The case is impossible.

(5) If $(a, b) \in \leq_{Q},(b, a) \in \leq_{Q}$, then $a=b$.

The cases (4), (6), (7), (9) are also impossible.

(II) $P$ is an ideal of $(V, \leq)$. In fact, let $a \in P$ and $V \ni b \leq a$. Then $b \in P$. Indeed, since $b \leq a$, we have $(b, a) \in \leq_{P},(b, a) \in \leq_{Q}$, or $(b, a) \in \bar{r}$. If $(b, a) \in \leq_{P}$, then $b \in P$. If $(b, a) \in \leq_{Q}$, then $a \in P \cap Q$. The case is impossible. If $(b, a) \in \bar{r}$, then, since $\bar{r} \subseteq P \times Q$, we have $a \in P \cap Q$. The case is impossible.

(III) The identity mapping

$$
i_{P}:\left(P, \leq_{P}\right) \longrightarrow(P, \leq \cap(P \times P)) \mid a \longrightarrow a
$$

is one-to-one and onto. Moreover, we have

$$
\leq_{P}=\leq \cap(P \times P) .
$$


Indeed, clearly, $\leq_{P} \subseteq \leq \cap(P \times P)$. Let $(a, b) \in \leq \cap(P \times P)$. Since $(a, b) \in \leq$, we have $(a, b) \in$ $\leq_{P},(a, b) \in \leq_{Q}$, or $(a, b) \in \bar{r}$. If $(a, b) \in \leq_{Q}$, then $a \in P \cap Q$, which is impossible. If $(a, b) \in \bar{r}(\subseteq P \times Q)$, then $b \in P \cap Q$ which is impossible. Thus we have $(a, b) \in \leq_{P}$.

By (2.4), the mapping $i_{P}$ is isotone and reverse isotone. Thus we have

$$
(P, \leq \cap(P \times P)) \approx\left(P, \leq_{P}\right) .
$$

We have $(V \backslash P, \leq \cap(V \backslash P \times V \backslash P)) \approx\left(Q, \leq_{Q}\right)$. Since $P \cap Q=\varnothing$ and $V=P \cup Q$, we have $V \backslash P=Q$. Moreover, the mapping

$$
i_{Q}:\left(Q, \leq_{Q}\right) \rightarrow(Q, \leq \cap(Q \times Q)) \mid a \rightarrow a
$$

is an isomorphism.

The converse statement is as follows: let $\left(P, \leq_{P}\right),\left(Q, \leq_{Q}\right)$ be ordered sets, $P \cap Q=\varnothing$, and $\left(V, \leq_{V}\right)$ an extension of $P$ by $Q$. Then there exist an ideal $P^{\prime}$ of $V$ and mappings

$$
\begin{aligned}
\varphi:\left(P, \leq_{P}\right) & \rightarrow\left(P^{\prime}, \leq_{V} \cap\left(P^{\prime} \times P^{\prime}\right)\right), \\
\psi:\left(Q, \leq_{Q}\right) & \rightarrow\left(V \backslash P^{\prime}, \leq_{V} \cap\left(\left(V \backslash P^{\prime}\right) \times\left(V \backslash P^{\prime}\right)\right)\right)
\end{aligned}
$$

which are isomorphisms.

Let $r \subseteq P \times Q$ such that for the set $\bar{r}$, we have $\bar{r}=\left\{(a, b) \in P \times Q \mid \varphi(a) \leq_{V} \psi(b)\right\}$.

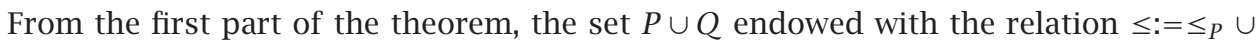
$\leq_{Q} \cup \bar{r}$ is an ordered set. We consider the mapping

$$
f:(P \cup Q, \leq) \longrightarrow\left(V, \leq_{V}\right) \mid a \longrightarrow f(a):= \begin{cases}\varphi(a) & \text { if } a \in P, \\ \psi(a) & \text { if } a \in Q\end{cases}
$$

The mapping $f$ is clearly well defined. Moreover, the following hold.

(I) The mapping $f$ is one-to-one. Let $a, b \in P \cup Q, f(a)=f(b)$. If $a, b \in P$, then $f(a):=\varphi(a), f(b):=\varphi(b), \varphi(a)=\varphi(b)$, and, since $\varphi$ is one-to-one, we have $a=b$. Let $a \in P, b \in Q$. Then $f(a):=\varphi(a) \in P^{\prime}, f(b):=\varphi(b) \in V \backslash P^{\prime}$. The case is impossible. The case $a \in Q, b \in P$ is also impossible. If $a, b \in Q$, then $\psi(a)=\psi(b)$ and $a=b$.

(II) $f$ is onto (since $\varphi$ and $\psi$ are onto).

(III) $f$ is isotone. Let $a, b \in P \cup Q, a \leq b$. Then $f(a) \leq_{V} f(b)$. Let $a \leq_{P} b$. Since $\varphi$ is isotone, we have $(\varphi(a), \varphi(b)) \in \leq_{V} \cap\left(P^{\prime} \times P^{\prime}\right) \subseteq \leq_{V}$. Since $a, b \in P$, we have $f(a):=\varphi(a), f(b):=\varphi(b)$. Then $(f(a), f(b)) \in \leq_{V}$, that is, $f(a) \leq_{V} f(b)$.

Let $a \leq_{Q} b$. Since $\psi$ is isotone, we have

$$
(\psi(a), \psi(b)) \in \leq_{V} \cap\left(\left(V \backslash P^{\prime}\right) \times\left(V \backslash P^{\prime}\right)\right) \subseteq_{V} .
$$

Since $a, b \in Q$, we have $f(a):=\psi(a), f(b):=\psi(b)$. Then $(f(a), f(b)) \in \leq_{V}$, that is, $f(a) \leq_{V} f(b)$.

Let $(a, b) \in \bar{r}$. By hypothesis, $(a, b) \in P \times Q$ and $\varphi(a) \leq_{V} \psi(b)$. Since $a \in P$, $b \in Q$, we have $f(a):=\varphi(a), f(b):=\psi(b)$. Then $f(a) \leq_{V} f(b)$. 
(IV) Let $a, b \in P \cup Q, f(a) \leq_{V} f(b)$. Then $a \leq b$. Indeed,

(1) let $a, b \in P$; then $f(a):=\varphi(a) \in P^{\prime}, f(b):=\varphi(b) \in P^{\prime}$, and $\varphi(a) \leq_{V} \varphi(b)$. Since $(\varphi(a), \varphi(b)) \in \leq_{V} \cap\left(P^{\prime} \times P^{\prime}\right)$ and $\varphi$ is reverse isotone, we have $(a, b) \in$ $\leq_{P} \subseteq \leq$, so $a \leq b$.

(2) Let $a \in P, b \in Q$. Then $f(a):=\varphi(a), f(b):=\psi(b), \varphi(a) \leq_{V} \psi(b)$. Since $(a, b) \in P \times Q$ and $\varphi(a) \leq_{V} \psi(b)$, we have $(a, b) \in \bar{r} \subseteq \leq$.

(3) Let $a \in Q, b \in P$. Then $f(a):=\psi(a) \in V \backslash P^{\prime}, f(b):=\varphi(b) \in P^{\prime}$. Since $V \ni f(a) \leq_{V} f(b) \in P^{\prime}$ and $P^{\prime}$ is an ideal of $V$, we have $f(a) \in P^{\prime}$. The case is impossible.

(4) Let $a, b \in Q$. Then $f(a):=\psi(a) \in V \backslash P^{\prime}, f(b):=\psi(b) \in V \backslash P^{\prime}$, and $\psi(a) \leq_{V}$ $\psi(b)$. Since $(\psi(a), \psi(b)) \in \leq_{V} \cap\left(\left(V \backslash P^{\prime}\right) \times\left(V \backslash P^{\prime}\right)\right)$ and $\psi$ is reverse isotone, we have $(a, b) \in \leq_{Q} \subseteq \leq$.

REMARK 2.2. Let $\left(P, \leq_{P}\right),\left(Q, \leq_{Q}\right)$ be ordered sets, $P \cap Q=\varnothing,\left(V, \leq_{V}\right)$ an extension of $P$ by $Q$, and $r \subseteq P \times Q$. From the first part of Theorem 2.1, the set $P \cup Q$ endowed with the relation $\leq:=\leq_{P} \cup \leq_{Q} \cup \bar{r}$, where $\bar{r}:=\left\{(a, b) \in P \times Q \mid \exists\left(a^{\prime}, b^{\prime}\right) \in r\right.$ such that $a \leq_{P} a^{\prime}$, $\left.b^{\prime} \leq_{Q} b\right\}$ is an ordered set. We remark that the mapping in (2.8) is an isomorphism if and only if $\bar{r}=\left\{(a, b) \in P \times Q \mid \varphi(a) \leq_{V} \psi(b)\right\}$.

"IF" PART. Let $f$ be an isomorphism. Let $(a, b) \in \bar{r}$. Since $a \leq b$, we have $f(a) \leq_{V}$ $f(b)$. Since $(a, b) \in P \times Q$, we have $f(a):=\varphi(a), f(b):=\psi(b)$. Then $\varphi(a) \leq_{V} \psi(b)$. Let $(a, b) \in P \times Q, \varphi(a) \leq_{V} \psi(b)$. Since $a \in P, b \in Q$, we have $f(a):=\varphi(a), f(b):=\psi(b)$. Then $f(a) \leq_{V} f(b)$ and $a \leq b$. Then we have $a \leq_{P} b, a \leq_{Q} b$, or $(a, b) \in \bar{r}$. If $a \leq_{P} b$, then $b \in P \cap Q$, which is impossible. If $a \leq_{Q} b$, then $a \in P \cap Q$, which is impossible. Thus we have $(a, b) \in \bar{r}$.

“ONLY IF” PART. Compare the proof of Theorem 2.1.

REMARK 2.3. If $\bar{r}=\varnothing$, then Theorem 2.1 is still valid. In the proof of the second part of Theorem 2.1, condition (2) in (IV) is an impossible case. Let $\bar{r}=\varnothing, a \in P, b \in Q$, $f(a) \leq_{V} f(b)$. Since $a \in P, b \in Q$, we have $f(a):=\varphi(a), f(b):=\psi(b)$. Then $\varphi(a) \leq_{V}$ $\psi(b)$. Since $(a, b) \in P \times Q, \varphi(a) \leq_{V} \psi(b)$, we have $(a, b) \in \bar{r}=\varnothing$, which is impossible.

If $r=\varnothing$, then $\bar{r}=\varnothing$, and the first part of Theorem 2.1 is valid. In that case, we have the trivial extension of $P$ by $Q$.

3. Some further results. The following question is natural: is there an $r \subseteq P \times Q$ such that $\bar{r}=\left\{(a, b) \in P \times Q \mid \varphi(a) \leq_{V} \psi(b)\right\}$ ? Under what conditions is that $r$ unique?

Proposition 3.1. Let $\left(V, \leq_{V}\right)$ be an extension of $P$ by $Q$. If

$$
r=\left\{(a, b) \in P \times Q \mid \varphi(a) \leq_{V} \psi(b)\right\}
$$

then $\bar{r}=r$.

Proof. First of all, $r \subseteq \bar{r}$. Now, let $(a, b) \in \bar{r}$. Then $(a, b) \in P \times Q$ and there exists $\left(a^{\prime}, b^{\prime}\right) \in r$ such that $a \leq_{P} a^{\prime}, b^{\prime} \leq_{Q} b$. Since $\left(a^{\prime}, b^{\prime}\right) \in r$, we have $\varphi\left(a^{\prime}\right) \leq_{V} \psi\left(b^{\prime}\right)$. Since $a \leq_{P} a^{\prime}$, we have $\left(\varphi(a), \varphi\left(a^{\prime}\right)\right) \in \leq_{V} \cap\left(P^{\prime} \times P^{\prime}\right) \subseteq \leq_{V}$, that is, $\varphi(a) \leq_{V} \varphi\left(a^{\prime}\right)$. Since 
$b^{\prime} \leq_{Q} b$, we have $\left(\psi\left(b^{\prime}\right), \psi(b)\right) \in \leq_{V} \cap\left(\left(V \backslash P^{\prime}\right) \times\left(V \backslash P^{\prime}\right)\right) \subseteq \leq_{V}$, that is, $\psi\left(b^{\prime}\right) \leq_{V} \psi(b)$. Since $(a, b) \in P \times Q$ and $\varphi(a) \leq_{V} \psi(b)$, we have $(a, b) \in r$.

Proposition 3.2. Let $V$ be an extension of $P$ by $Q$ and let $r=\{(a, b) \in P \times Q \mid \varphi(a)$ $\left.\leq_{V} \psi(b)\right\}$. The following are equivalent:

(1) the set $r$ is the unique subset of $P \times Q$ such that $\bar{r}=r$;

(2) if $(a, b) \in r$, then $a$ is minimal in $P$ and $b$ is maximal in $Q$.

Proof. $(1) \Rightarrow(2)$. Let $(a, b) \in r, a^{\prime} \leq_{P} a, b^{\prime} \geq_{Q} b, a^{\prime} \neq a, b^{\prime} \neq b$. Since $a^{\prime} \leq_{P} a$ and $\varphi$ is isotone, we have $\varphi\left(a^{\prime}\right) \leq_{V} \varphi(a)$. Since $b \leq_{Q} b^{\prime}$ and $\psi$ is isotone, we have $\psi(b) \leq_{V}$ $\psi\left(b^{\prime}\right)$. Since $(a, b) \in r$, we have $\varphi(a) \leq_{V} \psi(b)$. Since $\left(a^{\prime}, b^{\prime}\right) \in P \times Q$ and $\varphi\left(a^{\prime}\right) \leq_{V}$ $\psi\left(b^{\prime}\right)$, we have $\left(a^{\prime}, b^{\prime}\right) \in r$. We consider the set

$$
\rho:=r \backslash\left\{\left(a^{\prime}, b^{\prime}\right)\right\}
$$

Since $\left(a^{\prime}, b^{\prime}\right) \in r$ and $\left(a^{\prime}, b^{\prime}\right) \notin \rho$, we have $\rho \subset r(\subseteq P \times Q)$. We prove that $\bar{\rho}=r$ and get a contradiction.

Let $(x, y) \in \bar{\rho}$. Then there exists $\left(x^{\prime}, y^{\prime}\right) \in \rho$ such that $x \leq_{P} x^{\prime}, y^{\prime} \leq_{Q} y$. Since the mappings $\varphi$ and $\psi$ are isotone, we have $\varphi(x) \leq_{V} \varphi\left(x^{\prime}\right), \psi\left(y^{\prime}\right) \leq_{V} \psi(y)$. Since $\left(x^{\prime}, y^{\prime}\right) \in \rho(\subseteq r)$, we have $\varphi\left(x^{\prime}\right) \leq_{V} \psi\left(y^{\prime}\right)$. Since $(x, y) \in P \times Q$ and $\varphi(x) \leq_{V} \psi(y)$, we have $(x, y) \in r$.

Now, let $(x, y) \in r$. Then $(x, y) \in \bar{\rho}$. Indeed, since $(x, y),\left(a^{\prime}, b^{\prime}\right) \in r$, we have $(x, y)=$ $\left(a^{\prime}, b^{\prime}\right)$ or $(x, y) \neq\left(a^{\prime}, b^{\prime}\right)$. Let $(x, y)=\left(a^{\prime}, b^{\prime}\right)$. Since $(a, b) \in r,(a, b) \neq\left(a^{\prime}, b^{\prime}\right)$, we have $(a, b) \in \rho$. Since $\left(a^{\prime}, b^{\prime}\right) \in P \times Q,(a, b) \in \rho, a^{\prime} \leq_{P} a$, and $b^{\prime} \geq_{Q} b$, we have $\left(a^{\prime}, b^{\prime}\right) \in \bar{\rho}$. Then $(x, y) \in \bar{\rho}$.

Let $(x, y) \neq\left(a^{\prime}, b^{\prime}\right)$. Then, since $(x, y) \in r$, we have $(x, y) \in \rho \subseteq \bar{\rho}$.

(2) $\Rightarrow(1)$. Let $\rho \subseteq P \times Q$ such that $\bar{\rho}=r$. Then $\rho=r$. Indeed, first of all, $\rho \subseteq \bar{\rho}=r$. Let $(x, y) \in r(=\bar{\rho})$. Then there exists $(a, b) \in \rho(\subseteq \bar{\rho}=r)$ such that $x \leq_{P} a, b \leq_{Q} y$. Since $(a, b) \in r$, by hypothesis, $a$ is minimal in $P$ and $b$ is maximal in $Q$. Then $x=a, y=b$. Then $(x, y)=(a, b) \in \rho$.

The following proposition is useful for applications. It helps us to draw the figure of the extensions. As usual, for an ordered set $P$, we denote by " $\leq_{P}$ " (or " $\leq$ ") the order of $P$ and by " $\prec_{P}$ " (or " $\prec$ ") the covering relation of $P$. If $a_{x_{P}} b$ and $a \neq b$, we write $a<_{P} b$.

Proposition 3.3. Let $(V, \leq)$ be the extension of $\left(P, \leq_{P}\right)$ by $\left(Q, \leq_{Q}\right)$ constructed in the first part of Theorem 2.1. Then, for the covering relation " $\prec$ ” of $V$,

$$
\prec=\prec_{P} \cup \prec_{Q} \cup\left\{(a, b) \in r \mid \nexists\left(a^{\prime}, b^{\prime}\right) \in r, a \leq_{P} a^{\prime}, b^{\prime} \leq_{Q} b,\left(a^{\prime}, b^{\prime}\right) \neq(a, b)\right\} .
$$

Proof. Let $a \prec b$. Then $a<b$ and there does not exist $t \in V$ such that

$$
a<t<b \text {. }
$$

Since $a<b$, we have $a \leq_{P} b, a \neq b$ or $a \leq_{Q} b, a \neq b$ or $(a, b) \in \bar{r}$.

We note that if $(a, b) \in \bar{r}$, then $a \neq b$. This is because $\bar{r} \subseteq P \times Q, P \cap Q=\varnothing$. 
(1) Let $a<_{p} b$. Then $a \prec_{p} b$. Indeed, let $t \in P$ such that $a<_{p} t<_{p} b$. Since $(a, t) \in$ $<_{P} \subseteq<$, we have $a<t$. Since $(t, b) \in<_{P} \subseteq<$, we have $t<b$. Then $t \in V$ and $a<t<b$, which is impossible by (3.4).

(2) Let $a<_{Q} b$. As in the previous case, there does not exist $t \in Q$ such that $a<_{Q}$ $t<_{Q} b$. Thus $a \prec_{Q} b$.

(3) Let $(a, b) \in \bar{r}$.

First of all, we prove the following: if $\left(a^{\prime}, b^{\prime}\right) \in r, a \leq_{P} a^{\prime}, b^{\prime} \leq_{Q} b$, then

$$
\left(a^{\prime}, b^{\prime}\right)=(a, b) .
$$

Let $\left(a^{\prime}, b^{\prime}\right) \neq(a, b)$. If $a^{\prime} \neq a$, then $\left(a, a^{\prime}\right) \in<_{p} \subseteq<,\left(a^{\prime}, b^{\prime}\right) \in r \subseteq \bar{r} \subseteq \leq, a^{\prime} \neq b^{\prime}$, $\left(b^{\prime}, b\right) \in \leq_{Q} \subseteq \leq$, then $a<a^{\prime}<b^{\prime} \leq b$, where $a^{\prime} \in V$, which is impossible by (3.4). Similarly, if $b^{\prime} \neq b$, we get a contradiction.

Since $(a, b) \in \bar{r}$, there exists $\left(a^{\prime}, b^{\prime}\right) \in r$ such that $a \leq_{P} a^{\prime}, b^{\prime} \leq_{Q} b$. Then, by (3.5), $a^{\prime}=a, b^{\prime}=b$, so $(a, b) \in r$. Moreover, by (3.5), there is no $\left(a^{\prime}, b^{\prime}\right) \in r$ such that $a \leq_{P} a^{\prime}$, $b^{\prime} \leq_{Q} b,\left(a^{\prime}, b^{\prime}\right) \neq(a, b)$.

Conversely, let $a \prec_{p} b$. Then $a \prec_{b}$. Indeed, since $a \prec_{p} b$, we have $a<_{p} b$ and there does not exist $t \in P$ such that $a<_{p} t<{ }_{p} b$. Since $(a, b) \in<_{P} \subseteq<$, we have $a<b$. Let $t \in V$ such that $a<t<b$. Since $V \ni t<b \in P, P$ an ideal of $V$, we have $t \in P$. Since $(a, t) \in \leq \cap(P \times P)=\leq_{P}, a \neq t$, we have $a<_{P} t$. Since $(t, b) \in \leq \cap(P \times P)=\leq_{P}, t \neq b$, we have $t<_{P} b$. Then $a<_{P} t<_{P} b, t \in P$, which is impossible.

Let $a \prec_{Q} b$. Then $a \prec b$. Indeed, since $a \prec_{Q} b$, we have $a<_{Q} b$ and there is no $t \in Q$ such that $a<_{Q} t<_{Q} b$. Since $a<_{Q} b$, we have $a<b$. Let $t \in V$ such that $a<t<b$. If $t \in P$, then $V \ni a<t \in P$. Since $P$ is an ideal of $V$, we have $a \in P$, which is impossible. Thus $t \in Q$. We have $a<t<b ; a, t, b \in Q$. Then $a<_{Q} t<_{Q} b, t \in Q$, which is impossible.

Let $(a, b) \in r$ such that there is no $\left(a^{\prime}, b^{\prime}\right) \in r$ such that $a \leq_{P} a^{\prime}, b^{\prime} \leq_{Q} b,\left(a^{\prime}, b^{\prime}\right) \neq$ $(a, b)$. Then $a \prec b$. Indeed, since $(a, b) \in r$ and $r \subseteq \bar{r} \subseteq \leq$, we have $a \leq b$. Since $r \subseteq P \times Q$ and $P \cap Q=\varnothing$, we have $a \neq b$. Then $a<b$. Let $t \in V$ such that $a<t<b$. Then

(1) let $t \in P$. Since $t<b$, we have $t<_{P} b, t<_{Q} b$, or $(t, b) \in \bar{r}, t \neq b$. Then, since $(t, b) \in P \times Q$ and $P \cap Q=\varnothing$, we have $(t, b) \in \bar{r}$. Then there exists $\left(t^{\prime}, b^{\prime}\right) \in r$ such that $t \leq_{P} t^{\prime}, b^{\prime} \leq_{Q} b$. Since $a, t \in P, a<t$, we have $a<_{P} t$. Since $t^{\prime} \neq a$, we have $\left(t^{\prime}, b^{\prime}\right) \in r, a<_{P} t^{\prime}, b^{\prime} \leq_{Q} b,\left(t^{\prime}, b^{\prime}\right) \neq(a, b)$, which is impossible;

(2) let $t \in Q$. Since $a<t$, we have $a<_{p} t, a<{ }_{Q} t$, or $(a, t) \in \bar{r}, a \neq t$. Then $(a, t) \in \bar{r}$, so there exists $\left(a^{\prime}, t^{\prime}\right) \in r$ such that $a \leq_{p} a^{\prime}, t^{\prime} \leq_{Q} t$. Since $t, b \in Q, t<b$, we have $t<_{Q} b$. Since $t^{\prime} \neq b$, we have $\left(a^{\prime}, t^{\prime}\right) \in r, a<_{P} a^{\prime}, t^{\prime}<_{Q} b,\left(a^{\prime}, t^{\prime}\right) \neq(a, b)$, which is impossible.

4. Examples. We apply our results to the following examples. We always denote by $\mathbb{N}=\{1,2,3, \ldots\}$ the set of natural numbers and $a \mid b$ means $a$ divides $b$.

EXAMPLE 4.1. We consider the ordered sets $\left(P, \leq_{P}\right)$ and $\left(Q, \leq_{Q}\right)$ given below:

$$
P:=\{p \mid p \in \mathbb{N}, p \text { prime, } 1<p \leq 60 \text { or } p=1\} .
$$

That is,

$$
P=\{1,2,3,5,7,11,13,17,19,23,29,31,37,41,43,47,53,59\} .
$$


“ $\leq_{P}$ " is the order on $P$ defined by

$$
\leq_{P}:=\{(a, b) \in P \times P|a| b\} .
$$

We have

$$
\begin{aligned}
\leq_{P}=\{ & (1,1),(1,2),(1,3),(1,5),(1,7),(1,11),(1,13), \\
& (1,17),(1,19),(1,23),(1,29),(1,31),(1,37), \\
& (1,41),(1,43),(1,47),(1,53),(1,59),(2,2), \\
& (3,3),(5,5),(7,7),(11,11),(13,13),(17,17), \\
& (19,19),(23,23),(29,29),(31,31),(37,37), \\
& (41,41),(43,43),(47,47),(53,53),(59,59)\} .
\end{aligned}
$$

Let $Q:=\left\{2^{n} .23 \mid n \in \mathbb{N}\right\}$ and let " $\leq Q$ " be the order on $Q$ defined by

$$
\leq_{Q}:=\{(a, b) \in Q \times Q|a| b\} .
$$

We have

$$
2.23 \leq_{Q} 2^{2} .23 \leq_{Q} 2^{3} .23 \leq_{Q} \cdots \leq_{Q} 2^{n} .23 \leq_{Q} \cdots .
$$

We give the covering relation " $\prec$ " and $\left(P, \leq_{P}\right)$ is shown in Figure 4.1.

$$
\begin{gathered}
\prec=\{(1,2),(1,3),(1,5),(1,7),(1,11),(1,13),(1,17),(1,19),(1,23), \\
(1,29),(1,31),(1,37),(1,41),(1,43),(1,47),(1,53),(1,59)\} .
\end{gathered}
$$

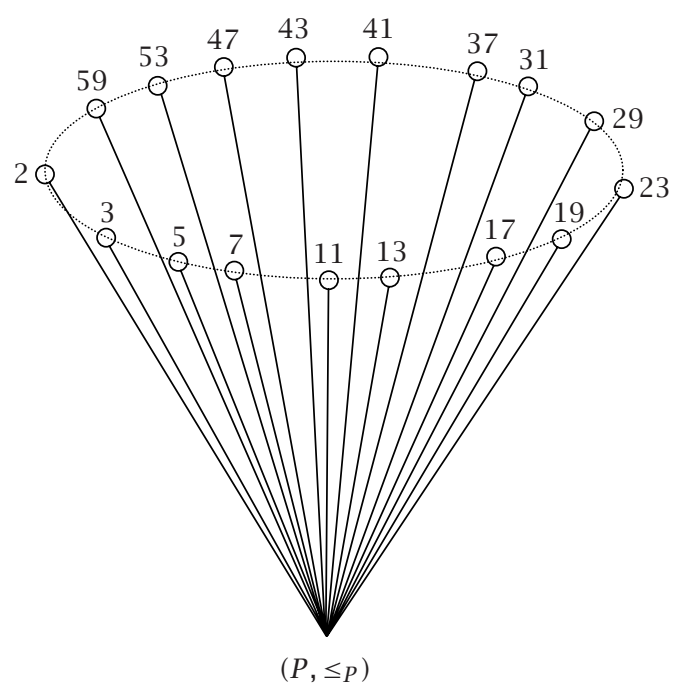

FIGURE 4.1 
$\left(Q, \leq_{Q}\right)$ is presented in Figure 4.2.

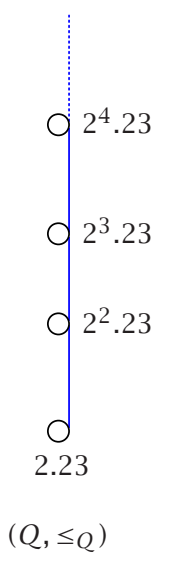

FIGURE 4.2

If $a \in P \cap Q$, then $a=1$ and $a=2^{n} .23$ for some $n \in \mathbb{N}$ or $a$ is a prime such that $a=2^{n} .23$ for some $n \in \mathbb{N}$, which is impossible. Thus the sets $P$ and $Q$ are disjoint.

Let $r:=\{(2,2.23),(23,2.23)\}(\subseteq P \times Q)$. From the first part of Theorem 2.1, the set $V:=P \cup Q$ endowed with the relation $\leq:=\leq_{P} \cup \leq_{Q} \cup \bar{r}$ is an ordered set and it is an extension of $P$ by $Q$.

The DeTERminAtion of $\bar{r}$. Let $(a, b) \in \bar{r}$. Then $(a, b) \in P \times Q$ and there exists $\left(a^{\prime}, b^{\prime}\right) \in r$ such that $a \leq_{P} a^{\prime}, b^{\prime} \leq_{Q} b$. Since $\left(a^{\prime}, b^{\prime}\right) \in r$, we have $\left(a^{\prime}, b^{\prime}\right)=(2,2.23)$ or $\left(a^{\prime}, b^{\prime}\right)=(23,2.23)$.

(A) Let $\left(a^{\prime}, b^{\prime}\right)=(2,2.23)$. Then $a^{\prime}=2, b^{\prime}=2.23$. Since $a \leq_{p} a^{\prime}=2$, we have $a=1$ or $a=2$. Since $2.23=b^{\prime} \leq_{Q} b$, we have $b=2^{n} .23$ for some $n \in \mathbb{N}$. Thus $(a, b)=\left(1,2^{n} .23\right)$ or $(a, b)=\left(2,2^{n} .23\right)$ for some $n \in \mathbb{N}$.

(B) Let $\left(a^{\prime}, b^{\prime}\right)=(23,2.23)$. Then $a^{\prime}=23, b^{\prime}=2.23$. Since $a \leq_{P} a^{\prime}=23$, we have $a=1$ or $a=23$. Since $2.23=b^{\prime} \leq_{Q} b$, we have $b=2^{n} .23$ for some $n \in \mathbb{N}$.

Thus $(a, b)=\left(1,2^{n} .23\right)$ or $(a, b)=\left(23,2^{n} .23\right)$ for some $n \in \mathbb{N}$. Hence we have

$$
\bar{r} \subseteq\left\{\left(1,2^{n} \cdot 23\right),\left(2,2^{n} \cdot 23\right),\left(23,2^{n} \cdot 23\right) ; n \in \mathbb{N}\right\}
$$

Since $\left(1,2^{n} .23\right) \in P \times Q,(2,2.23) \in r, 1 \leq_{P} 2,2.23 \leq_{Q} 2^{n} .23$, we have $\left(1,2^{n} .23\right) \in \bar{r}$ for every $n \in \mathbb{N}$. Since $\left(2,2^{n} .23\right) \in P \times Q,(2,2.23) \in r, 2 \leq_{P} 2,2.23 \leq_{Q} 2^{n} .23$, we have $\left(2,2^{n} .23\right) \in \bar{r}$ for every $n \in \mathbb{N}$. Similarly, $\left(23,2^{n} .23\right) \in \bar{r}$ for every $n \in \mathbb{N}$. Therefore, we have

$$
\bar{r}=\left\{\left(1,2^{n} .23\right),\left(2,2^{n} .23\right),\left(23,2^{n} .23\right) ; n \in \mathbb{N}\right\} .
$$


We give the covering relation “ $\prec$ " and $(V, \leq)$ is presented in Figure 4.3.

$$
\begin{aligned}
\prec=\{ & (1,2),(1,3),(1,5),(1,7),(1,11),(1,13),(1,17),(1,19),(1,23), \\
& (1,29),(1,31),(1,37),(1,41),(1,43),(1,47),(1,53),(1,59), \\
& (2,2.23),(23,2.23),\left(2.23,2^{2} .23\right),\left(2^{2} .23,2^{3} .23\right),\left(2^{3} .23,2^{4} .23\right), \ldots, \\
& \left.\left(2^{n-1} .23,2^{n} .23\right), \ldots\right\} .
\end{aligned}
$$

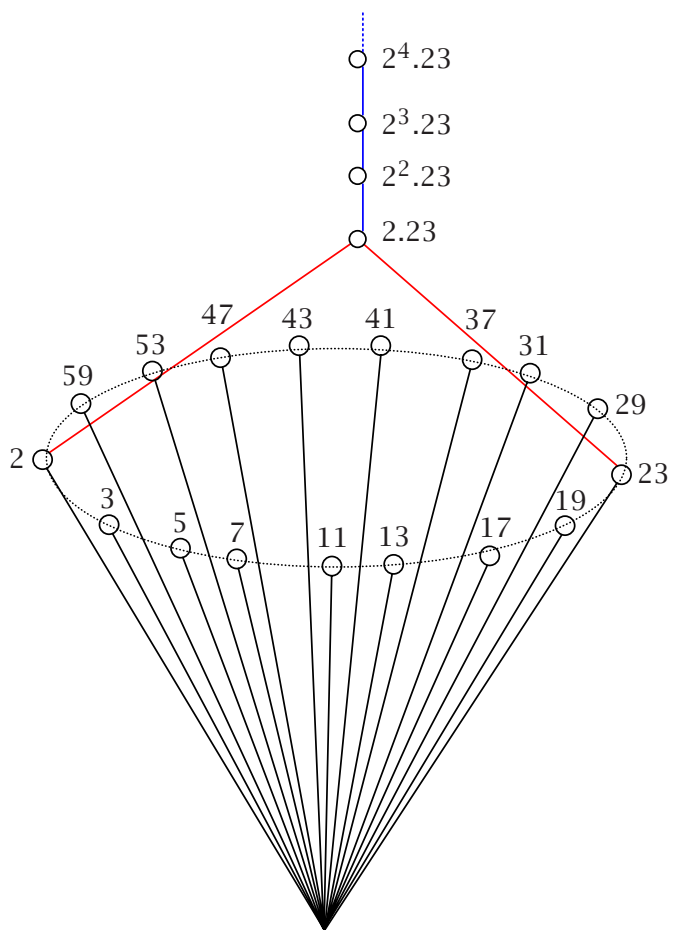

$\left(V, \leq_{V}\right)$

FIGURE 4.3

Note. For $a, b \in V$, we have $a \leq b$ if and only if $a \mid b$. Let $a, b \in V, a \leq b$. Since $\leq:=\leq_{P} \cup \leq_{Q} \cup \bar{r}$, we have $a, b \in P, a \mid b$ or $a, b \in Q, a \mid b$ or $(a, b) \in \bar{r}$. If $(a, b) \in \bar{r}$, then $(a, b)=\left(1,2^{n} .23\right)$ or $(a, b)=\left(2,2^{n} .23\right)$ or $(a, b)=\left(23,2^{n} .23\right)$ for some $n \in \mathbb{N}$. If $(a, b)=\left(1,2^{n} .23\right)$, then $a=1, b=2^{n} .23$, where $n \in \mathbb{N}$, then $a \mid b$. Similarly, if $(a, b)=$ $\left(2,2^{n} .23\right)$ or $(a, b)=\left(23,2^{n} .23\right)$, then $a \mid b$. Conversely, let $a, b \in V, a \mid b$. If $a, b \in P$, then $(a, b) \in \leq_{P} \subseteq \bar{r} \subseteq \leq$. Let $a \in P, b \in Q$, then $P \ni a \mid b=2^{n} .23$ for some $n \in \mathbb{N}$, thus $a=1$ or $a=2$ or $a=23$. If $a=1$, then $(a, b)=\left(1,2^{n} .23\right), n \in \mathbb{N}$, then $(a, b) \in \bar{r}$. Similarly, if $a=2$ or $a=23$, then $(a, b) \in \bar{r}$. Let $a \in Q, b \in P$. Then $2^{n} .23=a \mid b \in P$ for some $n \in \mathbb{N}$. The case is impossible. If $a, b \in Q$, then $(a, b) \in \leq_{Q} \subseteq \leq$.

As an application of the second part of Theorem 2.1, we give the following example. 
EXAMPLE 4.2. Let $\left(V, \leq_{V}\right),\left(P, \leq_{P}\right)$, and $\left(Q, \leq_{Q}\right)$ be the ordered sets defined by

$$
\begin{aligned}
V & =\{1,2,3,5,7,11,13,17,19,23,29,31,37,41,43,47,53,59\} \cup\left\{2^{n} .23 ; n \in \mathbb{N}\right\}, \\
\leq_{V} & :=\{(a, b) \in V \times V|a| b\}, \\
P & =\{1,2,3,5,7,11,13,17,19,23,29,31,37,41,43,47,53,59\}, \\
\leq_{P} & :=\{(a, b) \in P \times P|a| b\}, \\
Q & :=\left\{2^{n} .23 \mid n \in \mathbb{N}\right\}, \\
\leq_{Q} & :=\{(a, b) \in Q \times Q|a| b\} .
\end{aligned}
$$

Then

(1) $P$ is an ideal of $V$. Indeed, if $a \in P$ and $V \ni b \leq_{V} a$, then, since $b \mid a \in P$, we have $b=1$ or $b=a$, so $b \in P$;

(2) $V \backslash P=Q$.

Hence, $V\left(P, Q, i_{P}: P \rightarrow P, i_{Q}: Q \rightarrow V \backslash P\right)$ is an extension of $P$ by $Q$. Let $r$ be an arbitrary subset of $P \times Q$ such that

$$
\begin{aligned}
\bar{r} & =\left\{(a, b) \in P \times Q \mid i_{P}(a) \leq_{V} i_{Q}(b)\right\} \\
& =\left\{(a, b) \in P \times Q \mid a \leq_{V} b\right\} \\
& =\{(a, b) \in P \times Q|a| b\} .
\end{aligned}
$$

We have $\bar{r}=\left\{\left(1,2^{n} .23\right),\left(2,2^{n} .23\right),\left(23,2^{n} .23\right) ; n \in \mathbb{N}\right\}$. Indeed, let $(a, b) \in \bar{r}$. Then $(a, b) \in P \times Q$ and $a \mid b$. Since $P \ni a \mid b=2^{n} .23$, where $n \in \mathbb{N}$, we have $a=1$ or $a=2$ or $a=23$. Then $(a, b)=\left(1,2^{n} .23\right)$ or $(a, b)=\left(2,2^{n} .23\right)$ or $(a, b)=\left(23,2^{n} .23\right), n \in \mathbb{N}$.

On the other hand, since $\left(1,2^{n} .23\right) \in P \times Q$ and $1 \mid 2^{n} .23$, we have $\left(1,2^{n} .23\right) \in \bar{r}$ for every $n \in \mathbb{N}$. Since $\left(2,2^{n} .23\right) \in P \times Q$ and $2 \mid 2^{n} .23$, we have $\left(2,2^{n} .23\right) \in \bar{r}$ for every $n \in \mathbb{N}$. Similarly, $\left(23,2^{n} .23\right) \in \bar{r}$ for all $n \in \mathbb{N}$.

From the second part of Theorem 2.1, the set $P \cup Q$ endowed with the relation

$$
\leq:=\leq_{P} \cup \leq_{Q} \cup\left\{\left(1,2^{n} \cdot 23\right),\left(2,2^{n} \cdot 23\right),\left(23,2^{n} .23\right) ; n \in \mathbb{N}\right\}
$$

is an ordered set and $(P \cup Q, \leq) \approx\left(V, \leq_{V}\right)$.

We remark that for the set $r_{1}:=\{(a, b) \in P \times Q|a| b\}$ we have $\bar{r}_{1}=\{(a, b) \in P \times Q \mid$ $a \mid b\}$ (cf. Proposition 3.1).

As we have already seen, for the set $r_{2}:=\{(2,2.23),(23,2.23)\}$, we have

$$
\begin{aligned}
\bar{r}_{2} & =\left\{\left(1,2^{n} \cdot 23\right),\left(2,2^{n} \cdot 23\right),\left(23,2^{n} 2^{n} \cdot 23\right) ; n \in \mathbb{N}\right\} \\
& =\{(a, b) \in P \times Q|a| b\} .
\end{aligned}
$$

This example shows that the subset $r$ of $P \times Q$, for which $\bar{r}=\{(a, b) \in P \times Q \mid \varphi(a)$ $\left.\leq_{V} \psi(b)\right\}$ mentioned in the second part of Theorem 2.1, in general, is not uniquely defined. 
EXAMPLE 4.3. This is an example of extensions for which $\bar{r}=r$. Let $P:=\{2,3,5,7,210\}$. The set $P$ endowed with the relation $\leq_{P}:=\{(a, b) \in P \times P|a| b\}$ is an ordered set. $\left(P, \leq_{P}\right)$ is presented in Figure 4.4 .

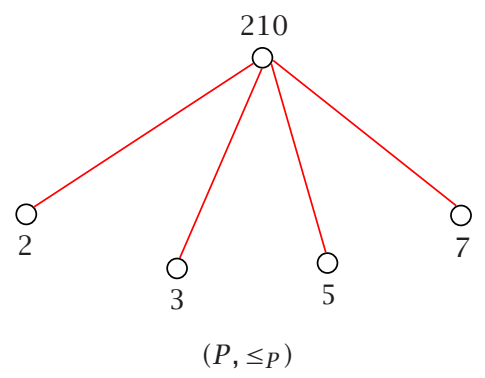

FIGURE 4.4

Let $Q:=\{11,22,33,55,77\}$. The set $Q$ with the relation

$$
\leq_{Q}:=\{(a, b) \in P \times P|a| b\}
$$

is an ordered set. $\left(Q, \leq_{Q}\right)$ is presented in Figure 4.5 .

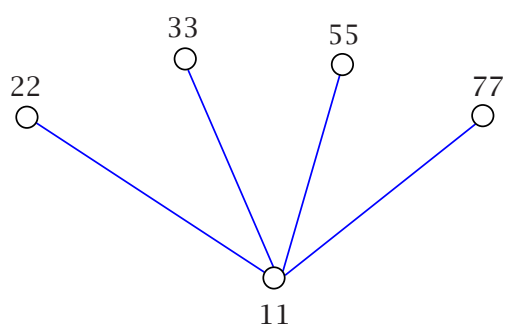

$\left(Q, \leq_{Q}\right)$

FIGURE 4.5

The ordered sets $P$ and $Q$ are disjoint. Let

$$
r:=\{(2,22),(3,33),(5,55),(7,77)\}(\subseteq P \times Q) .
$$

By the first part of Theorem 2.1, the set $V:=P \cup Q$ endowed with the relation $\leq:=\leq_{P} \cup$ $\leq_{Q} \cup \bar{r}$ is an ordered set and it is an extension of $P$ by $Q$.

For that extension, we have $\bar{r}=r$. Indeed, if $(a, b) \in \bar{r}$, then there exists $\left(a^{\prime}, b^{\prime}\right) \in r$ such that $a \leq_{P} a^{\prime}$ and $b^{\prime} \leq_{Q} b$. Since $\left(a^{\prime}, b^{\prime}\right) \in r$, we have $\left(a^{\prime}, b^{\prime}\right)=(2,22)$ or $\left(a^{\prime}, b^{\prime}\right)=$ $(3,33)$ or $\left(a^{\prime}, b^{\prime}\right)=(5,55)$ or $\left(a^{\prime}, b^{\prime}\right)=(7,77)$. If $\left(a^{\prime}, b^{\prime}\right)=(2,22)$, then $a^{\prime}=2, b^{\prime}=22$. Since $a \leq_{P} a^{\prime}=2$, we have $a=2$. Since $22=b^{\prime} \leq_{Q} b$, we have $b=22$. Thus we have $(a, b)=(2,22) \in r$. Similarly, if $\left(a^{\prime}, b^{\prime}\right)=(3,33),\left(a^{\prime}, b^{\prime}\right)=(5,55)$, or $\left(a^{\prime}, b^{\prime}\right)=(7,77)$, we have $(a, b) \in r$. Besides $r \subseteq \bar{r}$, so $r=\bar{r}$. 
The covering relation “ $\prec$ " of $(V, \leq)$ is shown as follows:

$$
\begin{gathered}
\prec=\{(2,210),(3,210),(5,210),(7,210),(11,22),(11,33),(11,55), \\
(11,77),(2,22),(3,33),(5,55),(7,77)\} .
\end{gathered}
$$

$(V, \leq)$ is presented in Figure 4.6.

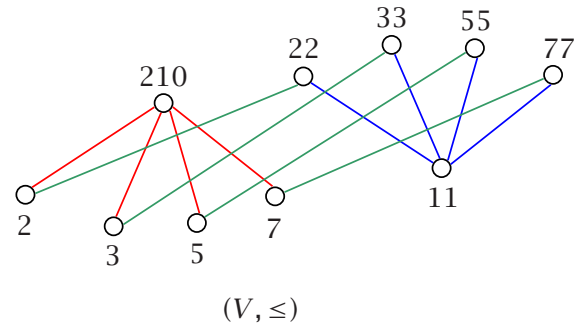

FIGURE 4.6

We have $r=\left\{(a, b) \in P \times Q \mid i_{P}(a)=a \leq_{V} b=i_{Q}(b)\right\}$. We remark that for each $(a, b) \in r$, the element $a$ is minimal in $P$ and $b$ is maximal in $Q$. According to Proposition 3.2 , the set $r$ is the unique element of $P \times Q$ such that $\bar{r}=r$.

EXAMPLE 4.4. We consider the ordered sets $P:=\{a, b, c, d, e\}$ and $Q:=\{x, y, k, l, m\}$ defined by Figures 4.7 and 4.8 , respectively. Let $r:=\{(a, y),(b, l),(c, m),(d, k)\}$. We have

$$
\bar{r}=\{(a, y),(a, k),(a, l),(a, m),(b, l),(c, k),(c, m),(d, k)\} .
$$

The covering relation of the extension $V:=L \cup K$ of $L$ by $K$ is shown as follows:

$$
\prec=\{(a, b),(a, y),(b, l),(c, d),(c, m),(d, k),(x, y),(y, k),(y, l),(y, m)\} .
$$

$V$ is presented in Figure 4.9 .

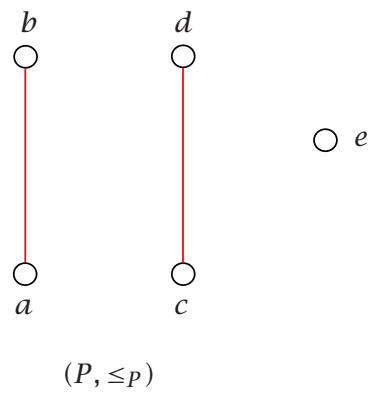

FIGURE 4.7 


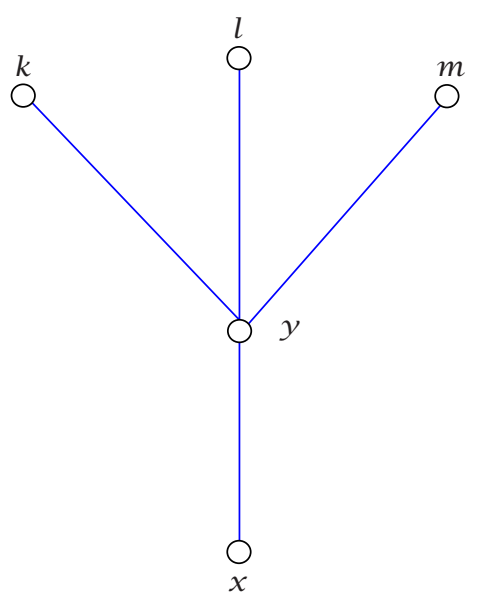

$\left(Q, \leq_{Q}\right)$

FIGURE 4.8

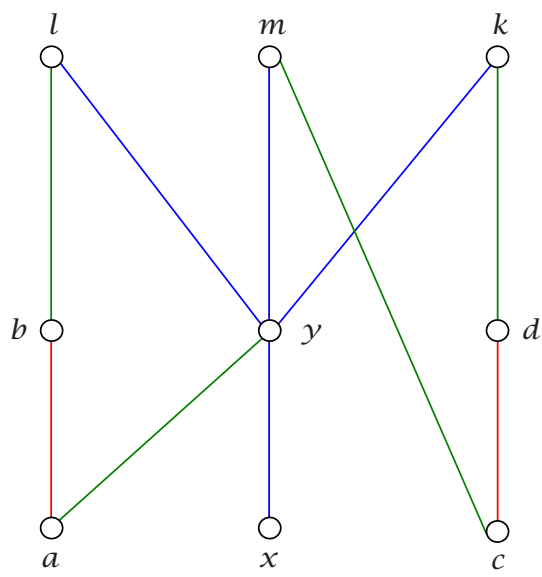

$(V, \leq)$

FIGURE 4.9

For the same sets $P$ and $Q$ we take $r:=\{(a, x),(b, l),(c, m),(d, k)\}$.

Then $\bar{r}=\{(a, x),(a, y),(a, k),(a, l),(a, m),(b, l),(c, k),(c, m),(d, k)\}$. The covering relation of the extension $V$ of $L$ by $K$ is shown as follows:

$$
\prec=\{(a, b),(a, x),(b, l),(c, d),(c, m),(d, k),(x, y),(y, k),(y, l),(y, m)\} .
$$

$V$ is presented in Figure 4.10.

Using computer, one can design and implement a program which gives all the (ideal) extensions of a finite ordered set $P$ by a finite ordered set $Q$. Again, using a program due to G. Lepouras, one can draw the figure of the ordered extension set. 


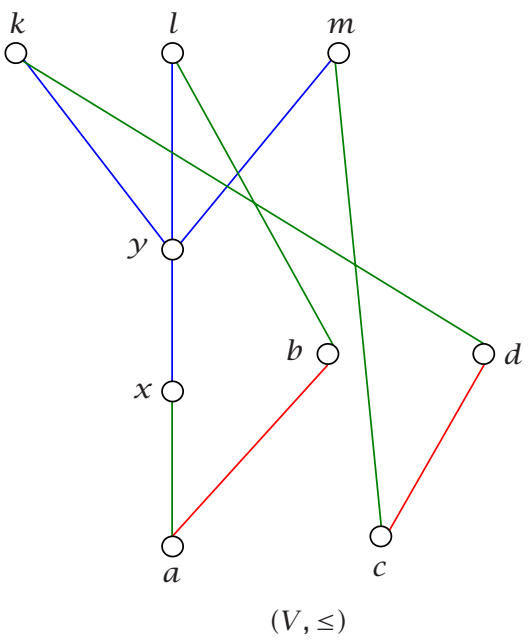

FIGURE 4.10

ACKNOWLEDGMENTS. This research has been supported by the Special Research Account of the University of Athens (Grant no. 70/4/5630). I would like to express my warmest thanks to the Managing Editor of the journal Professor Lokenath Debnath for his interest in my work and for communicating the paper.

\section{REFERENCES}

[1] G. Birkhoff, Lattice Theory, 3rd ed., American Mathematical Society Colloquium Publications, vol. 25, American Mathematical Society, Rhode Island, 1967.

[2] Fr. T. Christoph Jr., Ideal extensions of topological semigroups, Canad. J. Math. 22 (1970), $1168-1175$.

[3] A. H. Clifford, Extensions of semigroups, Trans. Amer. Math. Soc. 68 (1950), 165-173.

[4] A. H. Clifford and G. B. Preston, The Algebraic Theory of Semigroups. Vol. I, Mathematical Surveys, no. 7, American Mathematical Society, Rhode Island, 1964.

[5] J. A. Hildebrant, Ideal extensions of compact reductive semigroups, Semigroup Forum 25 (1982), no. 3-4, 283-290.

[6] A. J. Hulin, Extensions of ordered semigroups, Semigroup Forum 2 (1971), no. 4, 336-342.

[7] _ Extensions of ordered semigroups, Czechoslovak Math. J. 26(101) (1976), no. 1, $1-12$.

[8] N. Kehayopulu and P. Kiriakuli, The ideal extensions of lattices, Simon Stevin 64 (1990), no. 1, 51-60.

[9] N. Kehayopulu and M. Tsingelis, Ideal extensions of ordered semigroups, Comm. Algebra 31 (2003), no. 10, 4939-4969.

[10] M. Petrich, Introduction to Semigroups, Merrill Research and Lecture Series, Charles E. Merrill Publishing, Ohio, 1973.

Niovi Kehayopulu: Department of Mathematics, University of Athens, Panepistimioupolis, 15784 Athens, Greece

E-mail address: nkehayop@cc.uoa.gr 


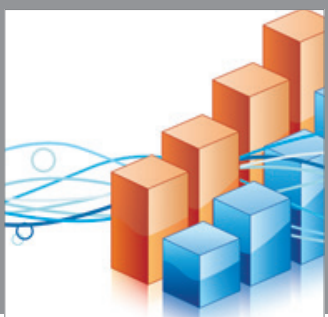

Advances in

Operations Research

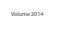

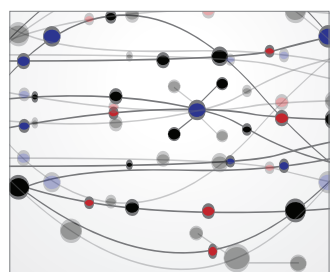

\section{The Scientific} World Journal
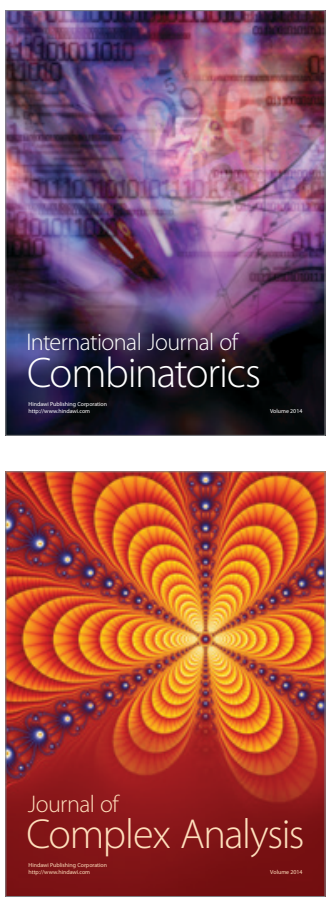

International Journal of

Mathematics and

Mathematical

Sciences
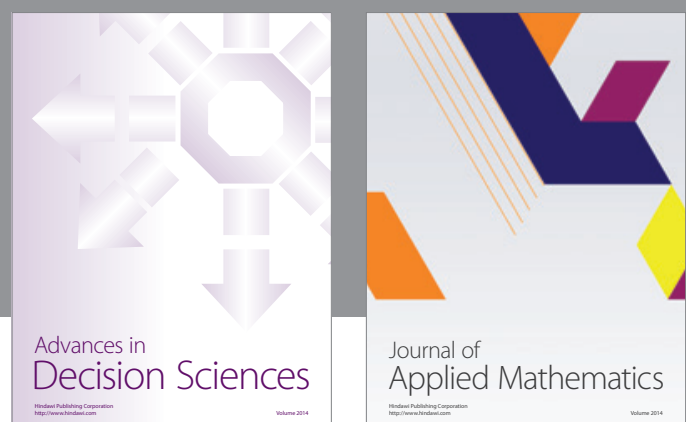

Journal of

Applied Mathematics
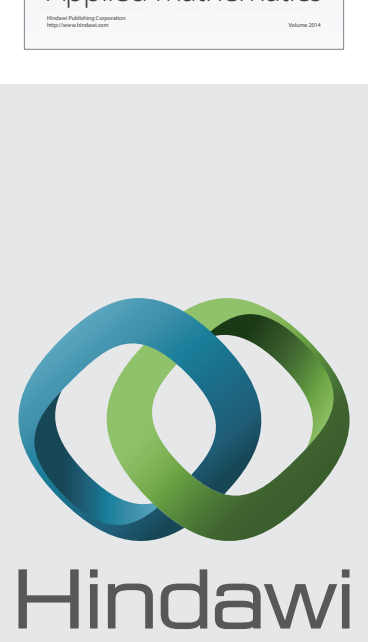

Submit your manuscripts at http://www.hindawi.com
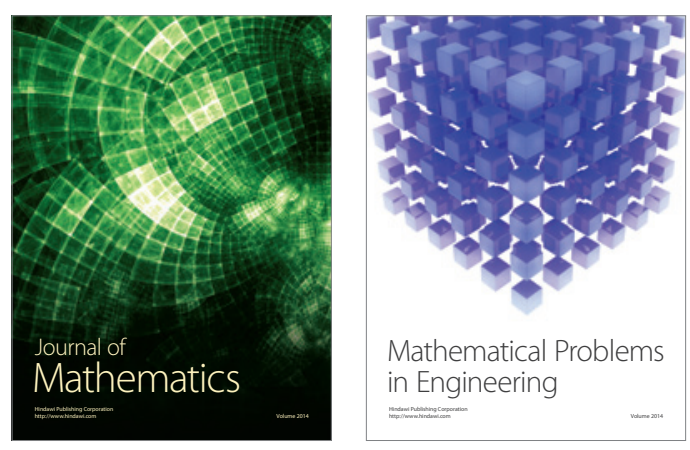

Mathematical Problems in Engineering
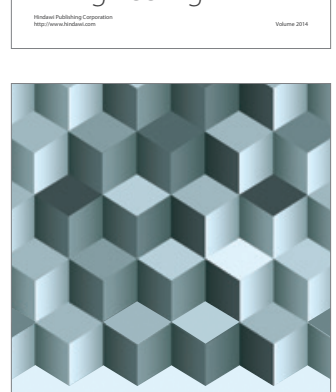

Journal of

Function Spaces
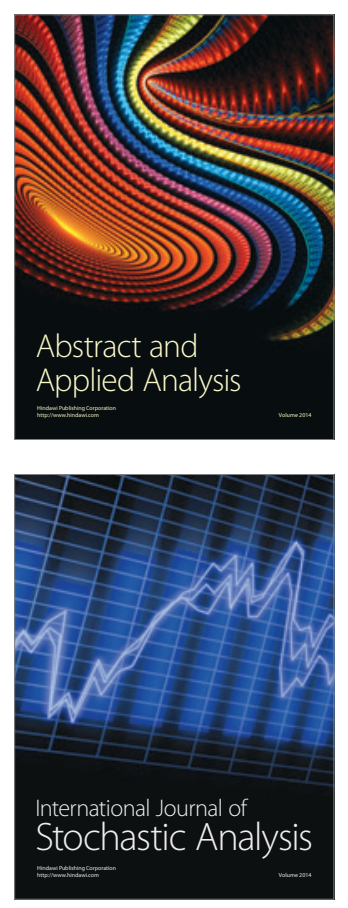

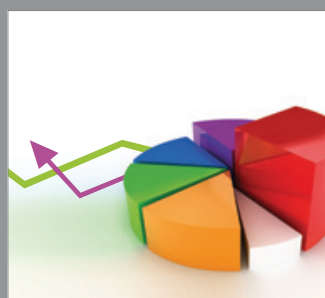

ournal of

Probability and Statistics

Promensencen
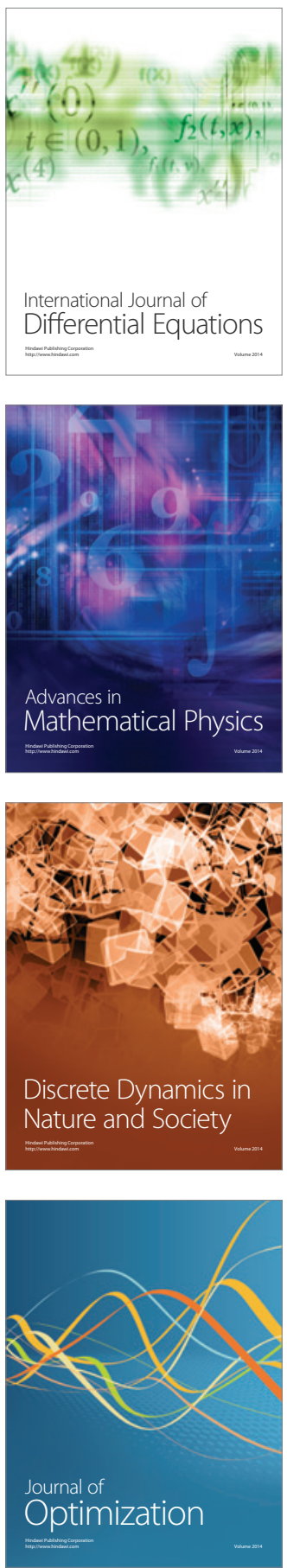